\title{
UNIFORMLY CONTINUOUS AND WEAKLY ALMOST PERIODIC FUNCTIONS ON SOME TOPOLOGICAL SEMIGROUPS
}

\author{
HENERI A. M. DZINOTYIWEYI
}

(Communicated by Jonathan M. Rosenberg)

\begin{abstract}
Let $\operatorname{LUC}(S)$ be the space of bounded left uniformly continuous functions and $\operatorname{WAP}(S)$ the space of bounded weakly almost periodic functions on a topological semigroup $S$. For a large class of topological semigroups called stips that are not relatively neocompact, we show that the quotient space $\operatorname{LUC}(S) /$ WAP $(S)$ contains an isometric linear copy of $l^{\infty}$. The result is particularly interesting on noting that the underlying topological semigroup is not assumed to support an absolutely continuous measure-a theme studied by the author in a previous paper.
\end{abstract}

Let $S$ be a locally compact Hausdorff topological semigroup, $C(S)$ the space of all bounded real-valued continuous functions on $S, \operatorname{LUC}(S):=\{f \in C(S)$ : the map $x \rightarrow{ }_{x} f$ of $S$ into $C(S)$ is norm continuous the space of left uniformly continuous functions on $S$ and $\operatorname{WAP}(S):=\{f \in C(S)$ : the set $\left\{{ }_{x} f: x \in S\right\}$ is relatively weakly compact $\}$ the space of weakly almost periodic functions on $S$. (Here the function ${ }_{x} f$ is given by ${ }_{x} f(y):=f(x y)$ for all $f \in C(S)$ and $x, y \in S$.) The space of right uniformly continuous functions, $\operatorname{RUC}(S)$, is the right-handed counterpart of $\operatorname{LUC}(S)$ and the space of uniformly continuous functions on $S$ is $\operatorname{UC}(S):=\operatorname{LUC}(S) \cap \operatorname{RUC}(S)$. If we use the weak topology in place of norm topology on $C(S)$, we obtain the weakly uniformly continuous functions $\mathrm{WUC}(S)$ in place of $\mathrm{US}(S)$.

For any subsets $A$ and $B$ of $S$ and points $x$ in $S$, we write

$$
\begin{aligned}
& A B:=\{a b: a \in A \text { and } b \in B\}, \\
& A^{-1} B:=\{y \in S: a y \in B \text { for some } a \in A\}, \\
& A^{-1} x:=A^{-1}\{x\} \text { and } x^{-1} A:=\{x\}^{-1} A .
\end{aligned}
$$

By symmetry the right-handed notation $B A^{-1}, x A^{-1}$, and $A x^{-1}$ can be easily defined. If $A_{1}, \ldots, A_{n}$ are subsets of $S$, we define

$$
\begin{aligned}
& A_{1} \otimes A_{2}:=\left\{A_{1} A_{2}, A_{1}^{-1} A_{2}, A_{1} A_{2}^{-1}\right\} \\
& A_{1} \otimes A_{2} \otimes A_{3}:=\left(U\left\{A_{1} \otimes B: B \in A_{2} \otimes A_{3}\right\}\right) \cup\left(U\left\{B \otimes A_{3}: B \in A_{1} \otimes A_{2}\right\}\right)
\end{aligned}
$$

Received by the editors July 23, 1990.

1980 Mathematics Subject Classification (1985 Revision). Primary 22A20; Secondary 43A15.

Key words and phrases. Topological semigroup, uniformly continuous functions, weakly almost periodic functions. 
and hence inductively similarly define $A_{1} \otimes A_{2} \otimes \cdots \otimes A_{n}$.

Following [2, 4], a subset $E$ of $S$ is said to be relatively neocompact if $E$ is contained in a union of sets in $A_{1} \otimes \cdots \otimes A_{n}$ for some compact subsets $A_{1}, \ldots, A_{n}$ of $S$.

We now define the underlying topological semigroup for our main result in this paper:

A locally compact topological semigroup $S$ with an identity element

1 is called stip if for each neighborhood $U$ of 1 we have that

$$
\begin{aligned}
& x \in \operatorname{int}\left(U^{-1}(U x) \cap(x U) U^{-1}\right) \quad \text { for all } x \in S, \text { and } \\
& 1 \in \operatorname{int}\left(U^{-1} u \cap v U^{-1}\right) \text { for some } u, v \in U .
\end{aligned}
$$

For an extensive study of stips, we refer the reader to $([4,6]$ and the references mentioned there). Stips form a very large class of topological semigroups many of which are known to support absolutely continuous measures (i.e. Radon measures on $S$ continuous under translation by points of $S$ )-see e.g. [4, 6].

In $[3,4]$ we showed that every nonrelatively neocompact topological semigroup $S$ admitting an absolutely continuous measure is such that the quotient space WUC $(S) /$ WAP $(S)$ contains an isometric linear copy of $l^{\infty}$ (and so is nonseparable)-thus solving a problem raised earlier and partially studied for topological groups by E. E. Granirer [5] and Ching Chou [1]. We also showed that for a stip $S, \operatorname{WAP}(S) \subseteq \mathrm{UC}(S)$ and $\operatorname{WUC}(S)=\mathrm{UC}(S)$ and raised the conjecture that $\operatorname{UC}(S) / \operatorname{WAP}(S)$ contains an isometric copy of $l^{\infty}$ whenever $S$ is not relatively neocompact. (Here $S$ is no longer assumed to support an absolutely continuous measure.) In this paper we solve the conjecture if the stip is assumed to be commutative or when $\operatorname{LUC}(S)$ is used in place of $\mathrm{UC}(S)$.

Theorem. Let $S$ be a stip that is not relatively neocompact. Then the quotient space $\operatorname{LUC}(S) / \mathrm{WAP}(S)$ contains an isometric linear copy of $l^{\infty}$.

Proof. From the proof of [3, Theorem 7.3, p. 234] we can find compact neighborhoods $V$ and $W$ of the identity, $1, w \in W$ and sequences $\left\{x_{n}\right\},\left\{y_{m}\right\}$ such that

$$
\left.\begin{array}{l}
V \subset W^{-1} w \cap w W^{-1} \cap W \\
x \in \operatorname{int}\left(w^{-1}(W x)\right) \text { for all } x \in S
\end{array}\right\}
$$

and

$$
\left.\begin{array}{l}
w^{-1}(W x) \cap V^{-1}\left(V x_{n} y_{m}\right) \neq \varnothing \text { implies } \\
\text { that } w^{-1}(W x) \cap V^{-1}\left(V x_{i} y_{j}\right)=\varnothing
\end{array}\right\}
$$

if any one of the following items holds:

(a) $n \leq m$ and $i>j$

(b) $n>m, i>j$, and $n \neq i$

(c) $n \leq m, i \leq j$, and $m \neq j$.

From [6, pp. 84-85] we can find a sequence of open neighborhoods of 1 , $\left\{V_{k}\right\}$, and a pseudometric $\rho$ such that

$$
\begin{aligned}
& V_{k+1}^{2} \subseteq V_{k} \subseteq V, \\
& V_{k+1}^{-1}\left(V_{k+1} x\right) \subseteq\left\{y \in S: \rho(y, x) \leq 2^{-k+1}\right\} \subseteq V_{k}^{-1}\left(V_{k} x\right), \\
& \rho(x z, y z) \leq \rho(x, y),
\end{aligned}
$$

for all $x, y, z \in S$ and $k \in \mathbb{N}$. 
We thus have

$$
\{y \in S: \rho(y, x) \leq 1\} \subseteq V^{-1}(V x) .
$$

Let $h_{n, m}$ be the function on $S$ given by

$$
h_{n, m}(x):=1-\min \left(\rho\left(x, x_{n} y_{m}\right), 1\right) .
$$

By (3) we have that

$$
\left\{x \in S: h_{n, m}(x) \neq 0\right\} \subseteq V^{-1}(V x) .
$$

Let $A_{k}:=\left\{x_{k_{n}}\right\}$ and $B_{k}:=\left\{y_{k_{m}}\right\}$ be subsequences of $\left\{x_{1}, x_{2}, \ldots\right\}$ and $\left\{y_{1}, y_{2}, \ldots\right\}$, respectively, constructed as done in [3, p. 228] and consider the function

$$
f_{k}:=\sum_{n=1}^{\infty} \sum_{n \leq m} h_{k_{n}, k_{m}}-\sum_{m=1}^{\infty} \sum_{m<n} h_{k_{n}, k_{m}} .
$$

From (2) and (4), we have that $0 \leq f_{k} \leq 1$.

Next we show that $f_{k} \in \operatorname{LUC}(S)$. To the latter end, let $\varepsilon>0$ be given. By the definition of a stip and the above choice of $\rho$, we can find an open neighborhood, 0 , of the identity such that

$$
0^{-1}(0 x) \subseteq w^{-1}(W x) \text { and } y \in 0^{-1}(0 x) \text { implies } \rho(x, y)<\varepsilon
$$

for all $x, y \in S$.

If both $f_{k}(x)$ and $f_{k}(y)$ equal zero, then $\left|f_{k}(x)-f_{k}(y)\right|<\varepsilon$. Now suppose $f_{k}(x) \neq 0$. Then $h_{k_{i}, k_{j}}(x) \neq 0$ for some $i, j \in \mathbb{N}$. By (4), (2), and the hypothesis on the choice of $A_{k}$ and $B_{k}$, we have

$$
h_{k_{n}, k_{m}}(x)=h_{k_{n}, k_{m}}(y)=0 \text { for those }(n, m) \text { in (5) such that }(n, m) \neq(i, j) \text {. }
$$

Hence

$$
\begin{aligned}
\left|f_{k}(x)-f_{k}(y)\right| & =\left|h_{k_{i}, k_{j}}(x)-h_{k_{i}, k_{j}}(y)\right| \\
& =\mid \min \left(\rho\left(x, x_{k_{i}} y_{k_{j}}\right), 1\right)-\min \left(\rho\left(y, x_{k_{i}} y_{k_{j}}\right), 1 \mid\right.
\end{aligned}
$$

We also note that

$$
h_{k_{i}, k_{j}}(x) \neq 0 \quad \text { implies } \rho\left(x, x_{k_{i}} y_{k_{j}}\right)<1 .
$$

So

$$
\begin{aligned}
& \left|f_{k}(x)-f_{k}(y)\right| \\
& \leq\left\{\begin{array}{l}
\left|\rho\left(x, x_{k_{i}} y_{k_{j}}\right)-\rho\left(y, x_{k_{i}} y_{k_{j}}\right)\right| \leq \rho(x, y)<\varepsilon \quad \text { if } \rho\left(y, x_{k_{i}} y_{k_{j}}\right) \leq 1 \\
\left|\rho\left(x, x_{k_{i}} y_{k_{j}}\right)-\left(\rho\left(x, x_{k_{i}} y_{k_{j}}\right)+\varepsilon\right)\right|=\varepsilon \quad \text { if } \rho\left(y, x_{k_{i}} y_{k_{j}}\right)>1,
\end{array}\right.
\end{aligned}
$$

since $\rho\left(y, x_{k_{i}} y_{k_{j}}\right) \leq \rho(y, x)+\rho\left(x, x_{k_{i}} y_{k_{j}}\right)$. Thus

$$
y \in 0^{-1}(0 x) \text { implies }\left|f_{k}(x)-f_{k}(y)\right| \leq \varepsilon \text { for all } x, y \in S .
$$

Since $y s \in 0^{-1}(0 x s)$ whenever $y \in 0^{-1}(0 x)$ for all $x$ and $y$ in $S$, we thus have that

$$
y \in 0^{-1}(0 x) \text { implies } \quad\left\|_{x}\left(f_{k}\right)-{ }_{y}\left(f_{k}\right)\right\|:=\sup \left\{\left|f_{k}(x s)-f_{k}(y s)\right|: s \in S\right\} \leq \varepsilon .
$$

Thus $f_{k} \in \operatorname{LUC}(S)$. 
By Grothendieck's characterisation of relatively weakly compact sets of functions, as similarly observed in [3], we have $f_{k} \notin \operatorname{WAP}(S)$ since

$$
f_{k}\left(x_{k_{i}} y_{k_{j}}\right)= \begin{cases}1 & \text { if } i \leq j, \\ -1 & \text { if } i>j .\end{cases}
$$
$l^{\infty}$

The rest of the proof now follows that of $[3, \S 4]$ to show that for each $\left(c_{k}\right) \in$

$$
\left(c_{k}\right) \rightarrow \sum_{k=1}^{\infty} c_{k} f_{k}
$$

yields an isometric linear copy of $l^{\infty}$ into $\operatorname{LUC}(S) / \mathrm{WAP}(S)$.

Remarks. 1. If $S$ is commutative then $\operatorname{LUC}(S)=\mathrm{UC}(S)$ and our theorem holds for uniformly continuous functions.

2. Let a stip $S$ be such that $C^{-1} D$ and $D C^{-1}$ are compact for all compact subsets $C$ and $D$. Then the relatively neocompact subsets of $S$ are precisely the relatively compact subsets. In particular if $S$ is not compact then $\mathrm{LUC}(S) / \mathrm{WAP}(S)$ contains an isometric linear copy of $l^{\infty}$, by our theorem.

3. Relatively neocompact stips can be very large. In fact we may still have $\operatorname{LUC}(S) / \mathrm{WAP}(S)$ containing an isometric linear copy of $l^{\infty}$ for certain relatively neocompact stips. For example

$$
S:=(\{0\} \times[0, \infty)) \cup\{(1,0)\} \text { with additive operation on }\{0\} \times[0, \infty)
$$

and

$$
x(1,0)=(1,0) x:=(1,0) \text { for all } x \in S,
$$

is a relatively neocompact stip. In fact $S=t^{-1} t$ where $t:=(1,0)$. But $H:=\{0\} \times[0, \infty)$ is a clopen nonrelatively neocompact stip contained in $S$. Hence our theorem can be applied to $H$ and trivially extend the result to $S$.

\section{ACKNOWLEDGMENT}

I am indebted to Professors J. F. Méla, C. C. Graham, and K. Keimel for their inspiring company when this research was being carried out. The research was supported by grants from SAREC and the University of Zimbabwe and was partially done at ICTP.

\section{REFERENCES}

1. Ching Chou, Weakly almost periodic functions and almost convergent functions on a group, Trans. Amer. Math. Soc. 206 (1975), 175-200.

2. H. A. M. Dzinotyiweyi, Algebras of measures on C-distinguished topological semigroups, Math. Proc. Cambridge Philos. Soc. 84 (1978), 323-336.

3. _ Nonseparability of quotient spaces of function algebras on topological semigroups, Trans. Amer. Math. Soc. 272 (1982), 223-235.

4. __ The analogue of the group algebra for topological semigroups, Pitman Adv. Publ. Prgm., Boston, London, Melbourne, 1984.

5. E. E. Granirer, Exposed points of convex sets and weak sequential convergence, Mem. Amer. Math. Soc., no. 123, Amer. Math. Soc., Providence, RI, 1972.

6. G. L. G. Sleijpen, Locally compact semigroups and continuous translations of measures, Proc. London. Math. Soc. (3) 37 (1978), 75-97. 